\title{
Dinâmica da produção e decomposição da serapilheira do araribá (Centrolobium tomentosum Guill. ex Benth. - Fabaceae) em uma mata ciliar, Rio Jacaré-Pepira, São Paulo
}

\author{
MARCOS P.M. AIDAR ${ }^{1,3}$ e CARLOS A. JOLY ${ }^{2}$
}

(recebido: 6 de fevereiro de 2002; aceito: 6 de março de 2003)

\begin{abstract}
Dynamics of litter production and decomposition of araribá (Centrolobium tomentosum Guill. ex Benth. Fabaceae) in a riparian forest, Jacaré-Pepira river, São Paulo). This study was conducted in a remaining riparian forest (42 ha) in the Jacaré-Pepira river, where Centrolobium tomentosum Guill. ex Benth., an early secondary and deciduous species, is the most important tree in the seasonal semideciduous forest. C. tomentosum's litter production reached $4.2 \mathrm{~kg}^{\text {ind }}{ }^{-1}$, with two peaks of leaf shedding (January/February $-25.1 \%$ and July/August $-52.3 \%$ ) representing an input to soil surface of (g.ind. ${ }^{-1}$ year $^{-1}$ ): $74.7 \mathrm{~N} ; 6.1 \mathrm{P} ; 29.0 \mathrm{~K} ; 73.9 \mathrm{Ca} ; 25.8 \mathrm{Mg}$ and 14.1 S. Leaf litter produced takes 19.5 months for decomposition, which is strongly seasonal. The quality and quantity of $C$. tomentosum's litter indicate its potentially important role in the functional and structural recovery of riparian forests in the São Paulo State.
\end{abstract}

Key words - Centrolobium tomentosum, Jacaré-Pepira River basin, litter production, seasonal semideciduous forest, transfer of nutrient

RESUMO - (Dinâmica da produção e decomposição da serapilheira do araribá (Centrolobium tomentosum Guill. ex Benth. - Fabaceae) em uma mata ciliar, Rio Jacaré-Pepira, São Paulo). O estudo foi realizado em um fragmento de mata ciliar (42 ha) onde a espécie arbórea, secundária inicial e decídua Centrolobium tomentosum Guill. ex Benth. é dominante no estrato superior da floresta estacional semidecidual. A espécie apresentou grande produção de serapilheira $\left(4,2 \mathrm{~kg}_{\text {.ind }}{ }^{-1}\right) \mathrm{com}$ um comportamento bimodal de deposição (janeiro/fevereiro - 25,1\% e julho/agosto - 52,3\%), e grande capacidade de transferência

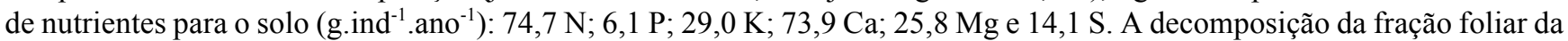
serapilheira apresentou padrão sazonal, resultando em cerca de 19,5 meses para seu desaparecimento. A qualidade e quantidade da serapilheira produzida por C. tomentosum indicam um alto potencial de utilização dessa espécie em programas de reabilitação funcional e estrutural das matas ciliares no Estado de São Paulo.

Palavras-chave - Bacia do rio Jacaré-Pepira, Centrolobium tomentosum, floresta estacional semidecidual, produção de serapilheira, transferência de nutrientes

\section{Introdução}

O estudo do fluxo dos nutrientes no ecossistema, isto é, a produção e decomposição da serapilheira com a conseqüente transferência destes para o ambiente, é essencial para a caracterização dos padrões de ciclagem (Golley 1983), pois representa a principal via de retorno de nutrientes e matéria orgânica à superfície do solo (Pagano \& Durigan 2000).

A mata ciliar devido à sua distribuição e importância peculiares, tem papel fundamental na estruturação e dinâmica das áreas de contato entre os ambientes terrestres e aquáticos continentais (Naiman et al. 1989, Naiman \& Déchamps 1990), mas apesar de

\footnotetext{
1. Instituto de Botânica, Seção de Fisiologia e Bioquímica de Plantas, Caixa Postal 4005, 01061-970 São Paulo, SP, Brasil.

2. Universidade Estadual de Campinas, Departamento de Botânica, Caixa Postal 6109, 13083-970 Campinas, SP, Brasil.

3. Autor para correspondência: maidar@uol.com.br
}

contar com proteção legal nos níveis Federal (Brasil 1965) e Estadual (São Paulo 1989), continua a ser devastada, principalmente sob pressão da expansão agro-industrial. Essas formações já não ocorrem mais de modo contínuo ao longo dos rios do Estado de São Paulo, sendo que as áreas remanescentes estão muito fragmentadas e sob forte pressão antrópica (Kronka et al. 1993).

Em vista desta gravíssima condição de preservação e da sua crescente degradação, com o conseqüente comprometimento da qualidade ambiental das bacias hidrográficas, é urgente o desenvolvimento de modelos para a recuperação das matas ciliares no Estado de São Paulo que considerem a reabilitação de suas características estruturais e funcionais, relacionadas principalmente à estabilidade do solo e margens dos cursos d'água, retenção de nutrientes e sedimentos, alta produtividade, hábitat para ocupação e regeneração de espécies e a regulação da qualidade e fluxo superficial da área (Rodrigues \& Gandolfi 2000). 
Existem poucas informações sobre a ciclagem de nutrientes em matas ciliares (Pagano \& Durigan 2000), pois poucos trabalhos foram desenvolvidos sobre o tema no Estado de São Paulo (Carpanezzi 1980, Dellitti 1984, Lamparelli 1983, Pagano \& Durigan 2000). Pouco se conhece também sobre a autoecologia das espécies arbóreas que ocorrem nas formações ciliares, sendo este um dos maiores obstáculos para a adequada implantação dos projetos de recuperação (Rodrigues \& Nave 2000).

Este trabalho avalia a quantidade e qualidade de serapilheira produzida por Centrolobium tomentosum Guill. ex Benth., visando verificar se a espécie pode ser considerada como alternativa para a recuperação funcional das formações ciliares do Estado de São Paulo.

\section{Material e métodos}

Descrição da área - O fragmento florestal estudado está localizado no Rio Jacaré-Pepira $\left(22^{\circ} 16^{\prime} \mathrm{S}\right.$ e $\left.48^{\circ} 10^{\prime} \mathrm{W}\right)$ a cerca de $8 \mathrm{~km}$ a jusante da cidade de Brotas, a uma altitude de 525-565 m, cuja composição florístico-fitossociológica foi estudada por Salis et al. (1994). A área tem cerca de 42 ha e constitui a maior mancha florestal ao longo de toda a calha principal do rio, cuja bacia drena cerca de $2.600 \mathrm{~km}^{2}$ (figura 1). O clima regional é classificado como Cwa (Köppen 1948), com temperatura média anual entre 21 e $23{ }^{\circ} \mathrm{C}$, média dos meses mais quentes entre 22 e $24^{\circ} \mathrm{C}$ e dos meses mais frios entre $18 \mathrm{e} 19^{\circ} \mathrm{C}$. A precipitação anual varia entre 1.300 e $1.500 \mathrm{~mm}$, com estação seca de abril a setembro (figura 2). Caracterização da espécie - O gênero Centrolobium tem dispersão limitada à América do Sul, e a espécie Centrolobium tomentosum Guill. ex Benth. é encontrada desde a Bahia até o Rio Grande do Sul, ocorrendo nas florestas úmidas (Floresta Ombrófila Densa), de planalto (Florestas Estacionais Semideciduais) e nas florestas de araucárias (Floresta Ombrófila Mista) (Bastos 1952, IBGE 1992). C. tomentosum é uma árvore decídua característica do estrato superior das florestas estacionais semideciduais do Estado de São Paulo, ocorrendo principalmente em áreas bem drenadas da mata ciliar (Aidar 1992, Joly et al. 2000). A espécie ocorre em pelo menos $70 \%$ das áreas de florestas estacionais investigadas no Estado de São Paulo (Salis et al. 1995), sendo considerada uma espécie de ampla distribuição nos domínios fitogeográficos do Estado de São Paulo

\section{BACIA DO RIO JACARÉ-PEPIRA}

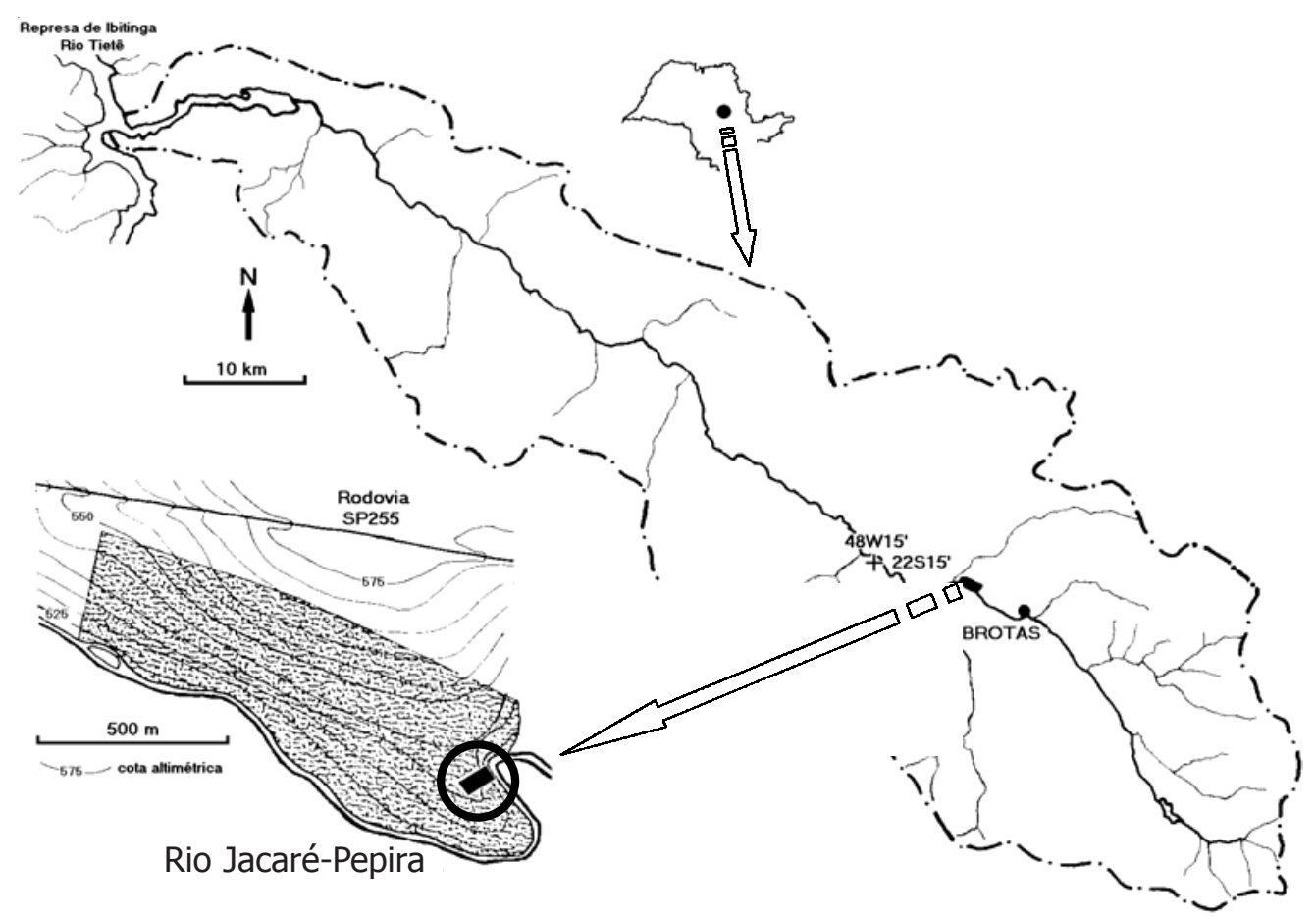

Figura 1. Localização da mata ciliar estudada na Bacia do Rio Jacaré-Pepira, Estado de São Paulo com o local de estudo assinalado (círculo).

Figure 1. Localization of riparian forest in the Jacaré-Pepira watershed, São Paulo State. The study site is indicated by a circle. 


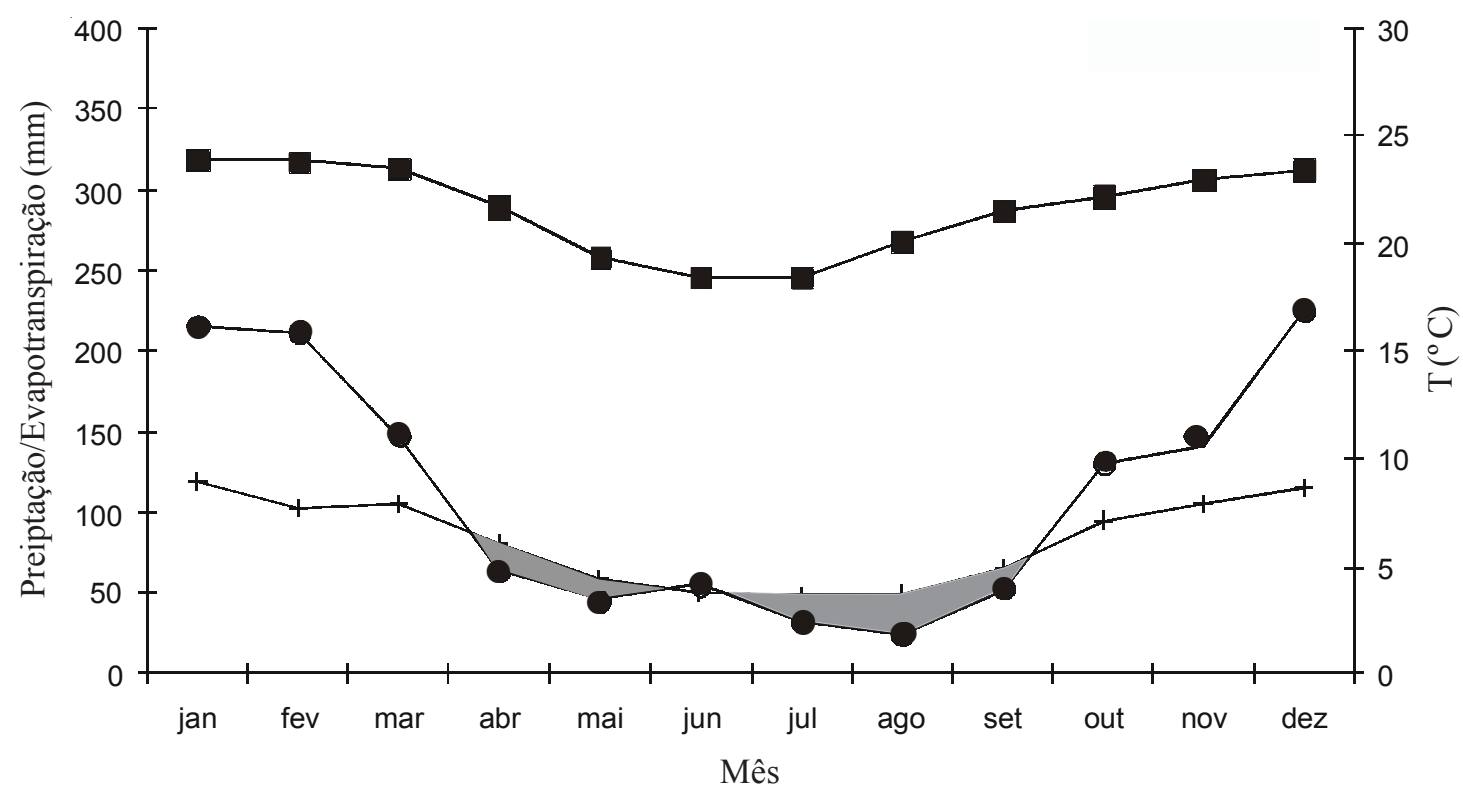

Figura 2. Balanço hídrico da região de estudo para o período 1956-1982. Precipitação de $1.345 \mathrm{~mm}$ e déficit hídrico de $42 \mathrm{~mm}$ (em cinza). Dados meteorológicos IAC - Jaú, latitude $22^{\circ} 17^{\prime} \mathrm{S}$, longitude $48^{\circ} 34^{\prime} \mathrm{W}$ e altitude $580 \mathrm{~m}$. ——-precipitação; — evapotranspiração; - —-temperatura.

Figure 2. Regional hydric balance for the period 1956-1982. Precipitation of $1.345 \mathrm{~mm}$ and hydric deficit of $42 \mathrm{~mm}$ (in gray). Meteorological data from Campinas Agronomic Institute (IAC), Jaú, latitude $22^{\circ} 17^{\prime} \mathrm{S}$, longitude $48^{\circ} 34^{\prime} \mathrm{W}$ and altitude $580 \mathrm{~m}$ a.s.l.——-precipitation; — - evapotranspiration; ——-temperature.

(Durigan et al. 2000). Na área de estudo, caracteriza-se como a espécie arbórea mais importante na estruturação do fragmento florestal, atingindo os maiores valores de IVI $(27,3)$ e IVC $(22,0)$ e densidade de 200 ind.ha $^{-1}$ (Salis et al. 1994). C. tomentosum apresenta um conjunto de características de desenvolvimento e regeneração que sugerem sua classificação como espécie secundária inicial (Aidar 1992, Gandolfi 2000), que apresenta crescimento relativamente rápido $\left(1 \mathrm{~m} \cdot \mathrm{ano}^{-1} \mathrm{em}\right.$ altura e $1 \mathrm{~cm} . \mathrm{ano}^{-1} \mathrm{em}$ diâmetro) com boa qualidade de madeira para usos diversos e grande potencial silvicultural (Bastos 1952). Apresenta também nodulação nas suas raízes e boa capacidade de absorção de nitrato (Aidar 1992).

Produção de serapilheira - Centrolobium tomentosum é uma espécie decídua que perde todas as suas folhas durante a estação seca. Assim, o acompanhamento da produção de serapilheira compreendeu o período de outubro de 1990 a outubro de 1991. A coleta dos dados foi realizada através de 10 coletores de $1 \mathrm{~m}^{2}$, confeccionados em metal e tela de nylon (malha $1 \mathrm{~mm}$ ), suspensos cerca de $20 \mathrm{~cm}$ do solo e dispostos em uma parcela de $100 \mathrm{~m}^{2}$ onde ocorriam 10 indivíduos adultos da espécie. Mensalmente as amostras coletadas foram submetidas a secagem a $60^{\circ} \mathrm{C}$ até peso constante e os coletores foram redispostos aleatoriamente dentro da parcela de $100 \mathrm{~m}^{2}$. As coletas foram separadas em folíolos, raques, flores e frutos. Os dados obtidos $\left(\mathrm{g} \cdot \mathrm{m}^{-2} \cdot \mathrm{dia}^{-1}\right)$ foram corrigidos para os valores médios por indivíduo $\left(\mathrm{g} . \mathrm{ind}^{-1}\right.$. $\left.\mathrm{ano}^{-1}\right)$ considerando a área total de amostragem de $100 \mathrm{~m}^{2}$.
Decomposição da fração foliar da serapilheira - O processo de decomposição foi acompanhado entre novembro 1989 e março 1991 através do uso de sacos de decomposição de $400 \mathrm{~cm}^{2}(20 \times 20 \mathrm{~cm})$ confeccionados com tela de nylon de malha $1 \mathrm{~mm}$. Estes foram dispostos em quatro locais escolhidos na área de levantamento fitossociológico (30 parcelas, $10 \times 10 \mathrm{~m}$ ) realizado por Salis et al. (1994), sendo cada conjunto composto por 36 sacos que continham $5 \mathrm{~g}$ de folíolos da espécie secos previamente em estufa a $60^{\circ} \mathrm{C}$. As coletas compostas de três sacos a cada ponto (12 sacos) foram realizadas mensalmente até julho de 1990, quando então o intervalo foi ampliado para dois meses. As análises químicas para quantificação dos macronutrientes nas amostras foram realizadas pelo laboratório Lagro (Laboratório Agronômico) de Campinas, através de metodologia padronizada (Zagatto et al. 1981). A amostra referente a março de 1991 foi descartada devido a acidente durante o transporte da coleta. A constante de decomposição foi calculada através da fórmula $k=-\operatorname{Ln}[1$ - (dPS/PSo)], onde dPS corresponde à perda de peso seco no período considerado e PSo ao peso seco inicial do período considerado (Olson 1963).

\section{Resultados}

A queda de serapilheira ocorreu durante todo o ano, com o mínimo no início da estação úmida (outubro 1990, com $0,3 \%$ do total da serapilheira produzida durante o 
período de estudo), e dois picos principais: um durante o verão, no auge da estação úmida (janeiro e fevereiro 1991 , com $25,1 \%$ do total de serapilheira), e um no fim do inverno, durante a estação seca (julho e agosto 1991, com 52,3\% do total da serapilheira) (figura 3). Estes picos apresentaram diferentes composições estruturais: o primeiro pico correspondeu a $20,3 \%$ do total de folíolos, $12,3 \%$ do total de raques e $93,7 \%$ do total de flores; o segundo pico correspondeu a $54,6 \%$ do total de folíolos, $63,0 \%$ do total de raques e $66,3 \%$ do total de frutos. A produção anual média por indivíduo atingiu $4,2 \mathrm{~kg}$, sendo que os folíolos representaram $63,2 \%$, as raques $19,0 \%$ (total de folhas $82,2 \%$ ), as flores $10,1 \%$ e os frutos $7,7 \%$ do total da serapilheira produzida durante o período de estudo.

A dinâmica sazonal das concentrações foliolares de nitrogênio e fósforo apresentou um comportamento bimodal inverso ao comportamento de queda das folhas, isto é, estas são maiores naqueles períodos onde a queda foi menor (setembro e novembro 1990; março e novembro 1991) e menores naqueles períodos de maior senescência foliar (janeiro/fevereiro e junho/agosto 1991). As concentrações foliolares de potássio e enxofre não apresentaram um padrão, enquanto as de cálcio, exceto nos folíolos jovens, e magnésio variaram pouco. Quanto às concentrações nas raques, o nitrogênio, fósforo e potássio apresentaram valores maiores nas estruturas jovens; o enxofre não variou; o cálcio e magnésio apresentaram valores maiores nas estruturas maduras (tabela 1). As flores apresentaram altos valores de potássio e os menores de cálcio, quando comparadas às outras estruturas. Os frutos maduros apresentaram maiores concentrações de potássio, enquanto os frutos abortados apresentaram maiores concentrações de nitrogênio, cálcio, magnésio e enxofre. As concentrações de fósforo não se alteraram ao longo do estudo (tabela 1). A transferência de elementos através da serapilheira resultou em (g.ind ${ }^{-1}$.ano $\left.{ }^{-1}\right): 74,7 \mathrm{~N} ; 6,1 \mathrm{P} ; 29,0 \mathrm{~K} ; 73,9$ $\mathrm{Ca} ; 25,8 \mathrm{Mg}$ e $14,1 \mathrm{~S}$.

O processo de decomposição apresentou padrão sazonal (figuras 4 e 5) com uma fase muito lenta durante a estação seca e mais fria (abril a setembro 1990). A avaliação do coeficiente de decomposição $(\mathrm{k})$ indicou que a taxa de decomposição em base anual foi de 44,2\% (figura 5). O tempo necessário para atingir 50\% de decomposição foi estimado através de ajuste de curva, resultando em 469 dias (15,6 meses). Segundo esta mesma aproximação, o desaparecimento foi atingido por

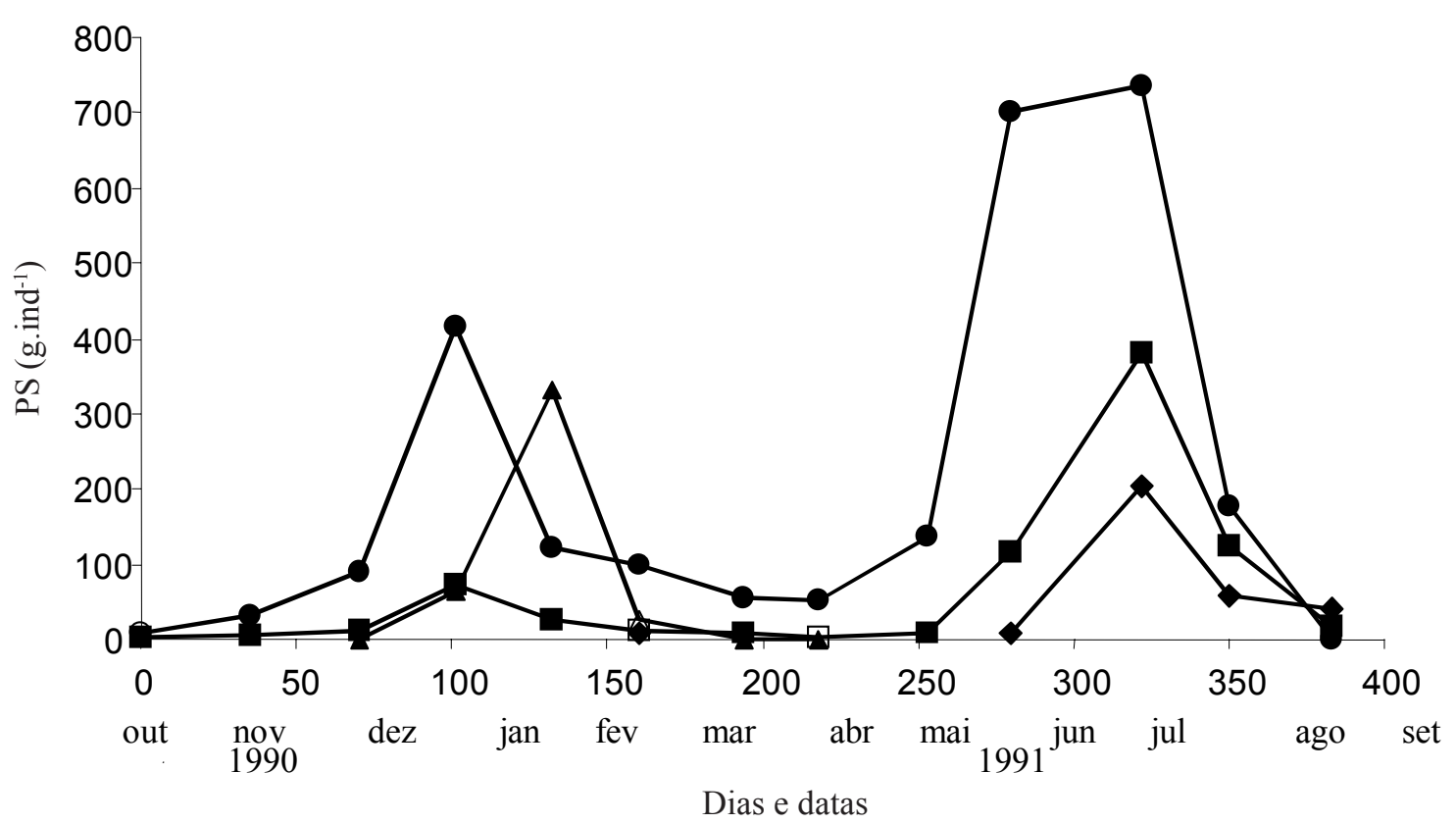

Figura 3 - Produção de serapilheira de Centrolobium tomentosum e suas frações principais ao longo do período de estudo (1990-1991) (g.ind $\left.{ }^{-1}\right)$. ——Folíolos; ———Raques; — _-Flores; ——_Frutos.

Figure 3. Litter production of Centrolobium tomentosum and its main fractions throughout the study period (1990-1991) (g.ind ${ }^{-1}$ ). ——Leaflets; —-—Rachis; —_—Flowers; ———Fruits. 
Tabela 1. Concentração de nutrientes (\% peso seco) no folhedo de Centrolobium tomentosum ao longo do período de estudo. Estado - estado de desenvolvimento de cada estrutura. Folíolos e Raques: imaturos - não totalmente expandidos; maduros totalmente expandidos. Flores: maduras - flores caídas após antese. Frutos: imaturos - frutos abortados logo após período de floração, não totalmente desenvolvidos; maduros - frutos totalmente desenvolvidos.

Table 1. Nutrient content (\% dry weight) in the litter of Centrolobium tomentosum through study period. State (estado) structure developmental stage; Leaflets (folíolos) and rachis (raques): immature (imaturos/imaturas) - not fully developed; mature (maduros/maduras) - fully developed; Flowers (flores): mature (maduras) - flowers dropped after anthesis. Fruits (frutos) - immature (imaturos) - aborted soon after flowering period, not fully developed; mature (maduros) - fully developed.

\begin{tabular}{llccrrrrr}
\hline Estrutura & Estado & Data & N & P & K & Ca & Mg \\
\hline Folíolos & maduros & set/90 & 2,43 & 0,16 & 0,42 & 1,87 & 0,33 & 0,19 \\
& maduros & nov/90 & 2,52 & 0,11 & 0,42 & 1,58 & 0,41 & 0,12 \\
& maduros & jan/91 & 1,85 & 0,07 & 0,22 & 1,61 & 0,30 & 0,14 \\
& maduros & fev/91 & 1,78 & 0,07 & 0,33 & 1,57 & 0,32 & 0,26 \\
& maduros & mar/91 & 2,10 & 0,09 & 0,25 & 1,86 & 0,31 & 0,19 \\
& maduros & jun/91 & 1,82 & 0,07 & 0,57 & 1,66 & 0,27 & 0,31 \\
& maduros & ago/91 & 1,69 & 0,07 & 0,58 & 1,95 & 0,27 & 0,34 \\
& imaturos & nov/91 & 2,72 & 0,32 & 1,63 & 1,02 & 0,31 & 0,26 \\
Raques & maduras & ago/90 & 0,87 & 0,06 & 0,55 & 2,6 & 0,48 & 0,19 \\
& imaturas & nov/91 & 2,53 & 0,34 & 1,5 & 0,93 & 0,23 & 0,19 \\
Flores & maduras & jan/91 & 1,76 & 0,32 & 1,43 & 0,74 & 0,26 & 0,17 \\
Frutos & imaturos & mar/91 & 2,29 & 0,16 & 0,39 & 1,22 & 0,27 & 0,21 \\
& maduros & ago/91 & 1,71 & 0,16 & 0,89 & 0,84 & 0,19 & 0,14 \\
\hline
\end{tabular}

volta de 584 dias (19,5 meses).

A liberação dos diferentes nutrientes da fração foliar da serapilheira para o ambiente (figura 6) revelou dinâmicas diversas: as concentrações de nitrogênio apresentaram um decaimento linear lento e constante,

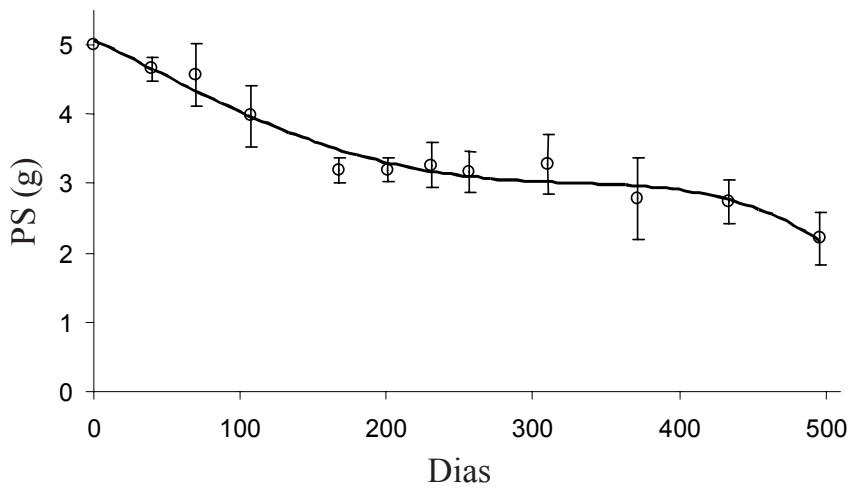

Figura 4. Curva de decomposição da fração foliar da serapilheira de Centrolobium tomentosum ( $\mathrm{g}$ Peso Seco; $\mathrm{n}=$ 12); $\mathrm{R}^{2}=0,966, \mathrm{p}<0,05$; ajuste polinomial $\mathrm{y}=2,10^{-10} \mathrm{x}^{4}+$ $2,10^{-7} x^{3}-2,10^{-5} x^{2}-0,01 x+5,0723$.

Figure 4. Decomposition curve for leaf litter of Centrolobium tomentosum (g Dry Weight; $\mathrm{n}=12$ ); $\mathrm{R}^{2}=0.966, \mathrm{p}<0.05$; polynomial fit $\mathrm{y}=2.10^{-10} \mathrm{x}^{4}+2 \cdot 10^{-7} \mathrm{x}^{3}-2 \cdot 10^{-5} \mathrm{x}^{2}-0.01 \mathrm{x}+5.0723$. sendo que o tempo necessário para a perda de $50 \%$ da quantidade inicial foi bastante alto (697 dias). As concentrações de cálcio apresentaram uma queda com padrão polinomial, consumindo 489 dias para perda de $50 \%$ da concentração inicial. O decaimento do potássio, do magnésio e do enxofre, apresentaram padrões logarítmicos. O potássio levou apenas 2,5 dias para atingir 50\% da concentração inicial, o magnésio parece estabilizar acima deste valor, enquanto que o enxofre levou 112 dias para atingir 50\% da concentração inicial. $\mathrm{O}$ fósforo apresentou comportamento que não configura uma curva de decaimento.

\section{Discussão}

A fenologia de Centrolobium tomentosum indica um pronunciado comportamento sazonal, caracterizado pela deciduidade e dispersão de frutos durante o inverno e floração no verão. Este padrão segue aqueles encontrados nas florestas sazonais de todas as regiões tropicais do mundo (Morellato et al. 1989), exceto quanto à floração durante o período úmido e a ocorrência de queda de folhas em dois períodos ao longo do ano, normalmente menos comum (Richards 1952, Rathcke \& Lacey 1985, Morellato 1992b). A dispersão 


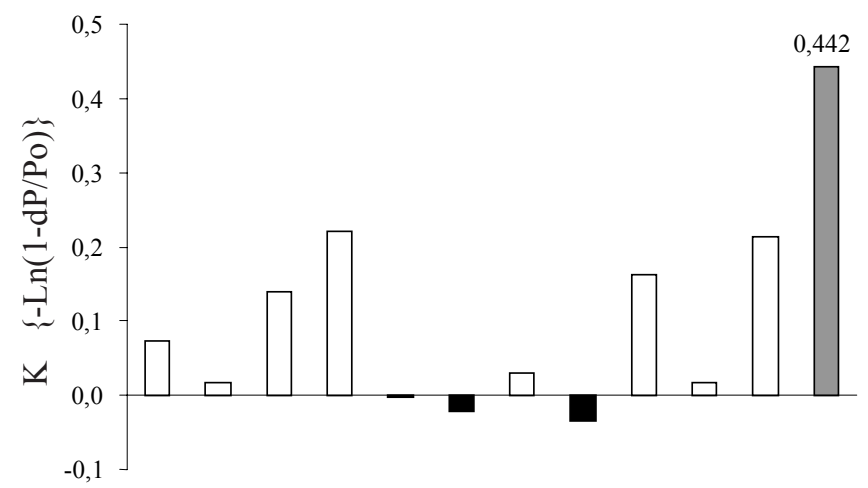

$\begin{array}{llllllllllll}40 & 70 & 107 & 167 & 201 & 231 & 257 & 310 & 372 & 434 & 496 & \text { total }\end{array}$ Dias

Figura 5. Constante de decomposição $(\mathrm{k})$ para cada intervalo de coleta ao longo do processo de decomposição das folhas de Centrolobium tomentosum na mata ciliar do Rio JacaréPepira.

Figure 5. Decomposition constant $(\mathrm{k})$ for each sampling interval throughout Centrolobium tomentosum's leaf litter decomposition in the riparian forest of Jacaré-Pepira river.

de frutos anemocóricos da espécie, do meio para o fim da estação seca, seria facilitada pela deciduidade (Janzen 1967, Frankie et al. 1974, Lieberman 1982, Morellato 1992a).

O comportamento decíduo da espécie durante o inverno (julho a setembro), evento bem documentado para outras espécies arbóreas características das matas ciliares do interior do Estado de São Paulo (Morellato et al. 1989, Pagano \& Durigan 2000), está relacionado ao estresse hídrico (Medina \& Klinge 1983, Pedroni et al. 2002). A sazonalidade é típica das florestas estacionais semidecíduas, formação que compõe a matriz vegetacional na qual se insere a mata ciliar em estudo (Rodrigues \& Nave 2000), onde boa parte das espécies derrubam suas folhas durante o período de seca, um padrão também associado à menor temperatura e fotoperíodo (Morellato 1992b). Entretanto, o pico observado no auge da estação quente e úmida (verão), imediatamente anterior a floração, é um evento menos comum em ecossistemas semelhantes, onde a antese das espécies ocorre principalmente no início da estação chuvosa (outubro a novembro) (Morellato 1992a, Carmo \& Morellato 2000, Morellato et al. 2000). A análise da concentração de nutrientes dos folíolos caídos ao longo do período de estudo indica a ocorrência de um processo de reabsorção dos nutrientes mais solúveis (nitrogênio, potássio, fósforo e enxofre) pré-antese, assim como acontece naqueles caídos durante a estação seca. Este comportamento parece estar associado à translocação destes nutrientes para investimento em outros processos: no verão, para investimento no esforço reprodutivo; no inverno, para futuro investimento na rebrota das folhas no início da estação úmida seguinte (Millard 1995). O processo de senescência foliar durante o verão poderia ainda auxiliar na atração de polinizadores, uma vez que a tonalidade amarelada adquirida por estas é muito semelhante a das flores (Aidar 1992).

A produção da fração foliar da serapilheira e a transferência de nutrientes de Centrolobium tomentosum para o solo foram mais elevados do que aqueles apresentados por Garrido \& Poggiani (1982) em Assis, SP, onde avaliaram a deposição anual de povoamentos puros de espécies nativas. Em relação à outras espécies estudadas, $C$. tomentosum contribuiu de 0,37 a 12 vezes mais $N$; de 2,7 a 9 vezes mais $P$; de 2 a 3 vezes mais $\mathrm{K}$, de 5 a 9 vezes mais $\mathrm{Ca}$; e de 4 a 19 vezes mais $\mathrm{Mg}$ (tabela 2).

A taxa de decomposição dos folíolos de C. tomentosum, em base anual (44,2\%), é comparável àquelas obtidas na Jamaica para Ilex obcordata Sw. e Podocarpus urbanii Pilg. e maior do que a de Guarea swartzii C. DC. (Tanner 1981). A dinâmica de decomposição apresentou um período de grande lentidão durante o inverno considerado como um reflexo direto do efeito regulatório dos fatores físicos ambientais, notadamente temperatura e umidade, sobre a atividade dos decompositores (Swift et al. 1979, Anderson \& Swift 1983). As informações na literatura sobre a produção e a decomposição de serapilheira de espécies arbóreas tropicais é muito escassa, sendo difícil a realização de outras comparações.

A dinâmica de transferência dos nutrientes da fração foliar da serapilheira em decomposição para o ambiente apresentou padrões diferentes. $\mathrm{O}$ nitrogênio sofreu decaimento constante sugerindo que está associado aos componentes estruturais do tecido vegetal. Também mostrou um efeito de elevação da concentração com o decorrer do processo de decomposição, sugerindo que tende a se tornar menos disponível, provavelmente devido à tanização de proteínas pelos fenóis produzidos na decomposição ou pela imobilização no protoplasma microbiano (Swift et al. 1979). O cálcio apresentou uma diminuição drástica na taxa de decaimento durante a estação seca, seguindo o padrão sazonal apresentado pelo processo de decomposição. O enxofre apresentou um decaimento logarítmico e um comportamento complexo quanto às concentrações nos tecidos durante o processo. Gosz et al. (1973) sugeriram que a retenção de enxofre deve 

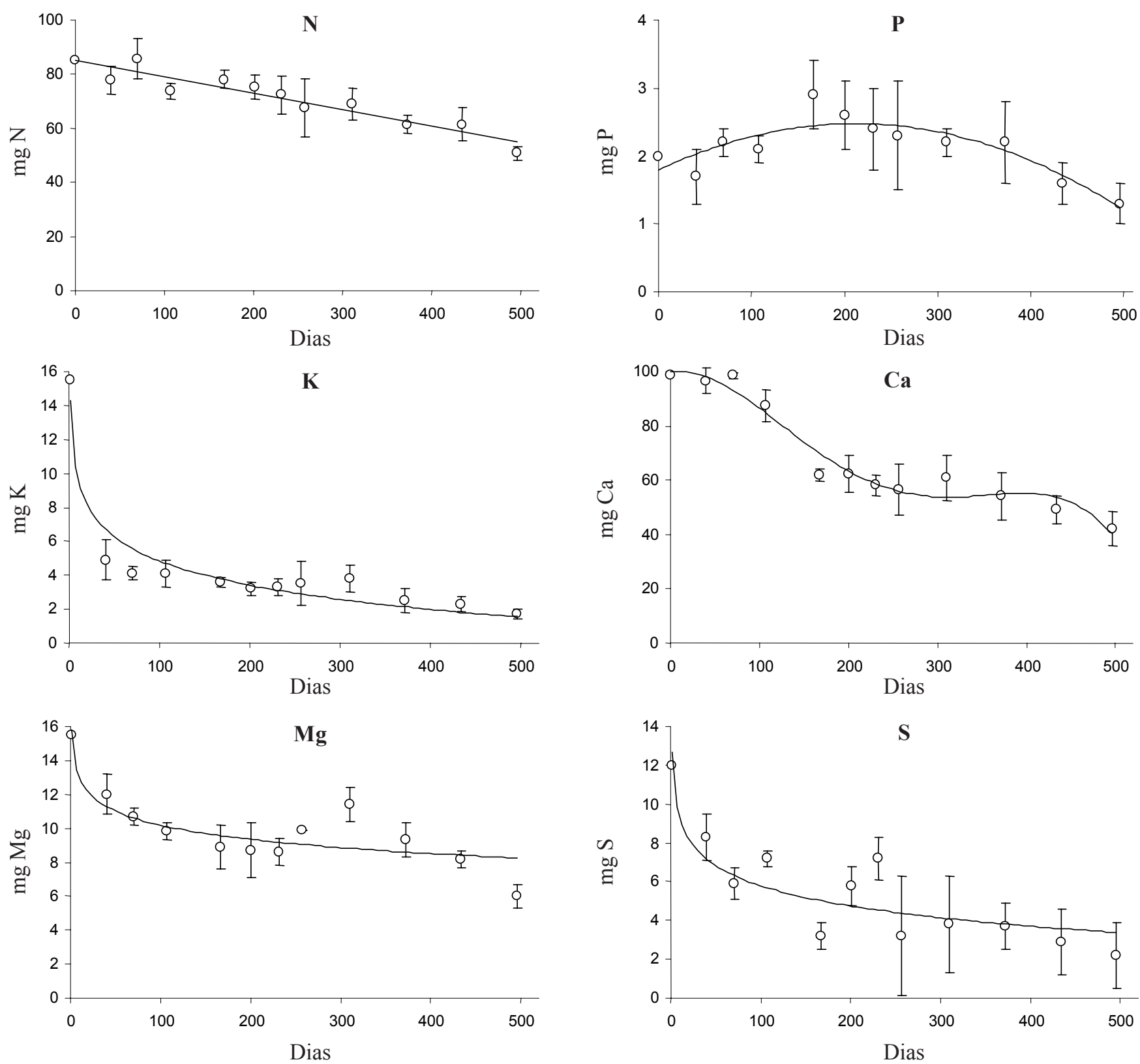

Figura 6. Curvas ajustadas de liberação dos macronutrientes da fração foliar da serapilheira de Centrolobium tomentosum para o solo $(\mathrm{n}=12)$. O conteúdo de nutrientes foi calculado utilizando-se o peso seco total das amostras a cada intervalo, assim a coleta inicial refere-se a mg de nutriente por $5 \mathrm{~g}$ de peso seco de folhas. O experimento foi iniciado em 10/11/89 e terminado em 21/03/91 (496 dias). Modelos ajustados: $\mathrm{N}$ - nitrogênio (linear) $\mathrm{y}=-0,061 \mathrm{x}+85,229, \mathrm{R}^{2}=0,892, \mathrm{p}<0,05 ; \mathrm{P}$ - fósforo (polinomial) $y=-1,510^{-5} \mathrm{x}^{2}+0,006 \mathrm{x}+1,792, \mathrm{R}^{2}=0,767, \mathrm{p}<0,05 ; \mathrm{P}$ - potássio (logarítmico) $\mathrm{y}=-2,055 \operatorname{Lnx}+14,3$, $\mathrm{R}^{2}=0,932, \mathrm{p}<0,05 ; \mathrm{Ca}$ - cálcio (polinomial) $\mathrm{y}=-10^{-8} \mathrm{x}^{4}+10^{-5} \mathrm{x}^{3}-0,003 \mathrm{x}^{2}+0,055 \mathrm{x}+99,809, \mathrm{R}^{2}=0,961, \mathrm{p}<0,05 ; \mathrm{Mg}-$ magnésio (logarítmico) $\mathrm{y}=-1,211 \mathrm{Lnx}+15,766, \mathrm{R}^{2}=0,758, \mathrm{p}<0,05 ; \mathrm{S}$ - enxofre (logarítmico) $\mathrm{y}=-1,501 \mathrm{Lnx}+12,701, \mathrm{R}^{2}$ $=0,788, \mathrm{p}<0,05$.

Figure 6. Fitted curves for macronutrient transference from Centrolobium tomentosum's leaf litter to soil $(\mathrm{n}=12)$. The nutrient content was calculated using the sample dry weight for each time interval. Initial dry weight was $5 \mathrm{~g}$. The experiment begun in $11 / 10 / 89$ and finished at 03/21/91 (496 days). Fitted models: $\mathrm{N}-$ nitrogen (linear) $\mathrm{y}=-0.061 \mathrm{x}+85.229, \mathrm{R}^{2}=0.892, \mathrm{p}<0.05 ; \mathrm{P}-$ phosphor (polynomial) $y=-1.510^{-5} \mathrm{x}^{2}+0.006 \mathrm{x}+1.792, \mathrm{R}^{2}=0.767, \mathrm{p}<0.05 ; \mathrm{P}-$ potassium (logarithmic) $\mathrm{y}=-2.055 \operatorname{Lnx}+14.3, \mathrm{R}^{2}$ $=0.932, \mathrm{p}<0.05 ; \mathrm{Ca}$ - calcium (polynomial) $\mathrm{y}=-10^{-8} \mathrm{x}^{4}+10^{-5} \mathrm{x}^{3}-0.003 \mathrm{x}^{2}+0.055 \mathrm{x}+99.809, \mathrm{R}^{2}=0.961, \mathrm{p}<0.05 ; \mathrm{Mg}$ - magnesium $\left(\right.$ logarithmic) $y=-1.211 \mathrm{Lnx}+15.766, \mathrm{R}^{2}=0.758, \mathrm{p}<0.05 ; \mathrm{S}$ - sulfur (logarithmic) $\mathrm{y}=-1.501 \mathrm{Lnx}+12.701, \mathrm{R}^{2}=0.788, \mathrm{p}<0.05$. 
Tabela 2. Produção da fração foliar da serapilheira de espécies arbóreas nativas e suas contribuições na transferência de nutrientes para o solo.

Table 2. Leaf fraction of litter production from native tree species and their contribution in the soil nutrient transfer.

\begin{tabular}{|c|c|c|c|c|c|c|c|}
\hline \multirow[t]{2}{*}{ Espécie } & \multirow[t]{2}{*}{ Família } & \multirow{2}{*}{$\begin{array}{l}\mathrm{kg} \cdot \text { ind }^{-1} \mathrm{ano}^{-1} \\
\text { Folhas }\end{array}$} & \multicolumn{5}{|c|}{ g.ind..$^{-1}$ ano $^{-1}$} \\
\hline & & & $\mathrm{N}$ & $\mathrm{P}$ & $\mathrm{K}$ & $\mathrm{Ca}$ & $\mathrm{Mg}$ \\
\hline Centrolobium tomentosum Guill. ex Benth. ${ }^{1}$ & Leguminosae & 3,43 & 54,3 & 2,9 & 14,2 & 67,0 & 23,0 \\
\hline Anadenanthera falcata (Benth.) Brenan ${ }^{2}$ & Leguminosae & 1,75 & 34,0 & 1,1 & 5,8 & 12,7 & 2,8 \\
\hline Astronium urundeuva (Fr. All.) Engl. ${ }^{2}$ & Anacardiaceae & 0,92 & 13,0 & 1,0 & 5,3 & 9,6 & 2,4 \\
\hline Tabebuia impetiginosa (Mart.) Standl. ${ }^{2}$ & Bignoniaceae & 0,39 & 4,5 & 0,3 & 4,2 & 7,8 & 1,2 \\
\hline
\end{tabular}

${ }^{1}$ Este estudo; ${ }^{2}$ Garrido \& Poggiani (1982), modificado para fins de comparação; espécies ocorrentes em matas ciliares do Brasil extraamazônico (Rodrigues \& Nave 2000).

${ }^{1}$ This study; ${ }^{2}$ Garrido \& Poggiani (1982), modified in order to compare; species occurring in the gallery forests form extra-Amazonian Brazil.

estar associada à sua imobilização no protoplasma dos organismos decompositores. O potássio e o magnésio também apresentaram decaimento logarítmico, com grande perda inicial através da lixiviação (Anderson \& Swift 1983), permanecendo então relativamente estáveis como resultado da retenção de potássio pelos organismos heterótrofos (Gosz et al. 1973) e da parede celular como pectato de magnésio (Swift et al. 1979). O fósforo apresentou um comportamento complexo de difícil compreensão, não caracterizando decaimento.

Pagano \& Durigan (2000) indicaram que a mata ciliar da Estação Experimental de Marília (solo úmido) apresenta produção de serapilheira diferenciada quando comparada às outras áreas estudadas em floresta estacional do interior do Estado de São Paulo, pois apresenta considerável produção de serapilheira durante o verão (estação úmida). Os autores relacionaram esse comportamento à maior ocorrência de espécies pioneiras e lianas na área, que normalmente apresentam maior produção de serapilheira em comparação com florestas mais maduras. Entretanto, os resultados apresentados pelos autores sugerem que o comportamento de deposição de serapilheira observado na área, provavelmente esteja associado à grande ocorrência de Centrolobium tomentosum, que representou $50 \%$ dos indivíduos (600 ind. ha ${ }^{-1}$ ) amostrados naquela formação florestal. Utilizando-se a produção de serapilheira obtida no presente estudo, i.e. $4,2 \mathrm{~kg}$ ind $^{-1} \cdot \mathrm{ha}^{-1} \cdot \mathrm{ano}^{-1}$, a espécie teria contribuído $\operatorname{com} 28,3 \%$ do total da serapilheira e $30,6 \%$ da fração foliar produzida anualmente no local. Os autores classificaram o fragmento florestal de Marília (solo úmido) em sexto lugar na transferência de nitrogênio para o solo, o que provavelmente está relacionado à alta qualidade da serapilheira de C. tomentosum, como demonstrado no presente estudo. Se utilizarmos novamente os parâmetros obtidos aqui, i.e.,

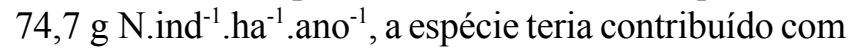
cerca de $24,2 \%$ do total de nitrogênio transferido para o solo no local.

Os resultados obtidos no presente trabalho quanto à quantidade e a qualidade da serapilheira produzida por Centrolobium tomentosum indicam uma grande contribuição dessa espécie na ciclagem de nutrientes na mata ciliar estudada e, potencialmente, em outras áreas similares nos Estados de São Paulo, Paraná, Minas Gerais e Mato Grosso do Sul. Os dados deste estudo também sugerem que a espécie tem boa capacidade de assimilação de nitrogênio do solo, configurando uma potencial capacidade de filtração do fluxo de água superficial e subsuperficial que atravessa o sistema ciliar.

A contaminação dos cursos d'água no interior do Estado de São Paulo com poluentes que contém elevadas concentrações de compostos nitrogenados, entre outros, originados de atividades agropecuárias (biocidas, fertilizantes, agroquímicos, resíduos, vinhaça, etc.) e urbanas (efluentes domésticos) (Cetesb 2002), poderia ser minimizada através da recuperação das áreas ciliares com espécies que apresentam maior capacidade de filtração dos fluxos superficiais e subsuperficiais, reestabelecendo a capacidade tamponante da vegetação ciliar e contribuindo no controle da qualidade da água de deflúvio na bacia hidrográfica (Lima \& Zakia 2000). Desse modo, pela sua capacidade de absorver $\mathrm{N}$ do solo e pela sua abundante produção de serapilheira de boa qualidade nutricional, a espécie Centrolobium tomentosum pode 
ser considerada como uma alternativa para utilização em projetos para a recuperação de matas ciliares no sudeste do Brasil, especialmente sobre solos distróficos e/ou esgotados pelo uso agro-industrial, pois apresenta características que favorecem a retenção de nutrientes e sedimentos, alta produtividade, e a regulação da qualidade e fluxo superficial da área.

Agradecimentos - O Projeto Jacaré-Pepira recebeu financiamento das seguintes instituições: Prefeitura Municipal de Brotas (SP), Ibama, CNPq (Processos 40.1297/88-8; 820945-87.0; 821117-88.2; 501450-91.2; 521566-93.2), da Finep (Processo 43.89.0478.00), Fapesp (Processo 91/1535-4; Faep/ Unicamp (Processos 028/88; 588/91; 284/92; 667/92), Grupo Pão de Açúcar, Conservation International e Unesco/MAB.

\section{Referências bibliográficas}

AIDAR, M.P.M. 1992. Ecologia do araribá (Centrolobium tomentosum Guill. ex Benth - FABACEAE) e o ecótono Mata Ciliar da bacia do rio Jacaré-Pepira, São Paulo. Dissertação de mestrado, Universidade Estadual de Campinas, Campinas.

ANDERSON, J.M. \& SWIFT, M.J. 1983. Decomposition in tropical forests. In Tropical rainforest: ecology and management (S.L. Sutton, T.C. Whitmore \& A.C. Chadwick, eds.). Blackwell Scientific Publ., London, p.287-310.

BASTOS, H.M. 1952. Contribuição para o conhecimento dendrológico das espécies do gênero Centrolobium. Rodriguésia 6:125-167.

BRASIL. 1965. Código Florestal. Lei Federal N. 4.771 de 1965. Imprensa Oficial do Brasil. (http://wwwt.senado.gov.br/ legbras/).

CARMO, M.R.B. \& MORELLATO, L.P.C. 2000. Fenologia de árvores e arbustos das Matas Ciliares da Bacia do Rio Tibagi, Estado do Paraná, Brasil. In Matas ciliares: conservação e recuperação (R.R. Rodrigues \& H.F. Leitão Filho, eds.). Editora da USP/Fapesp, São Paulo. p.125-141.

CARPANEZZI, A.A. 1980. Deposição de material orgânico e nutrientes em uma floresta natural e em uma plantação de eucaliptos no interior do Estado de São Paulo. Dissertação de mestrado, Universidade de São Paulo, Esalq, Piracicaba.

CETESB. 2002. Relatório de qualidade das águas interiores do Estado de São Paulo - 2001. Série Relatórios. Cetesb/SMA.

DELLITTI, W.B.C. 1984. Aspectos comparativos da ciclagem de nutrientes minerais na Mata Ciliar, no Campo Cerrado e na Floresta implantada de Pinus elliotti Engelm. var. elliotti (Mogi-Guaçu). Tese de doutorado, Universidade de São Paulo, São Paulo.
DURIGAN, G., RODRIGUES, R.R. \& SCHIAVINI, I. 2000. A heterogeneidade ambiental definindo a metodologia de amostragem da floresta ciliar. In Matas ciliares: conservação e recuperação (R.R. Rodrigues \& H.F. Leitão Filho, eds.). Editora da USP/Fapesp, São Paulo, p.159-167

FRANKIE, G.M., BAKER, H.G. \& OPLER, P.A. 1974. Comparative phenological studies of trees in tropical lowland wet and dry forest sites of Costa Rica. Journal of Ecology 62:881-913.

GANDOLFI, S. 2000. História natural de uma floresta estacional semidecidual no município de Campinas (São Paulo, Brasil). Tese de doutorado, Universidade Estadual de Campinas, Campinas.

GARRIDO, M.A.O. \& POGGIANI, F. 1982. Avaliação da quantidade e do conteúdo de nutrientes do folhedo de alguns povoamentos puros e mistos de espécies indígenas. Silvicultura em São Paulo 15/16:1-22.

GOLLEY, F.B. 1983. Tropical rain forest ecosystems: structure and function. Elsevier, Amsterdam.

GOSZ, J.R., LIKENS, G.E. \& BORMANN, F.H. 1973. Nutrient release from decomposing leaf and branch litter in the Hubbard Brook Forest, New Hampshire. Ecological Monographs 47:173-191.

IBGE. 1992. Manual técnico da vegetação brasileira. Manuais técnicos em geociências v.1, Instituto Brasileiro de Geografia e Estatística, Rio de Janeiro.

JANZEN, D.H. 1967. Synchronization of sexual reproduction of trees within the dry-season in Central America Evolution 21:620-637.

JOLY, C.A., SPIGOLON, J.R., LIEBERG, S.A., SALIS, S.M., AIDAR, M.P.M., METZGER, J.P.W., ZICKEL, C.S., LOBO, P.C., SHIMABUKURO, M.T., MARQUES, M.C.M. \& SALINO, A. 2000. Projeto Jacaré-Pepira - O desenvolvimento de um modelo de recomposição da Mata Ciliar com base na florísitca regional. In Matas ciliares: conservação e recuperação (R.R. Rodrigues \& H.F. Leitão Filho, eds.). Editora da USP/Fapesp, São Paulo, p.271-287

KRONKA, F.J.N., MATSUKUMA, C.K., NALON, M.A., CALI, I.H.D., ROSSI, M., MATTOS, I.F.A., SHIN-IKE, M.S. \& PONTINHA, A.A.S. 1993. Inventario Florestal do Estado de São Paulo. SMA/CINP/Instituto Florestal.

KÖPPEN, W. 1948. Climatologia. Ed. Fondo Cultura Economia, Mexico City.

LAMPARELLI, M.C. 1983. Aporte de serapilheira ao Rio Itaqueri (Itirapina, SP) e sua contribuição à economia de nutrientes. Dissertação de mestrado, Universidade Federal de São Carlos, São Carlos.

LIEBERMAN, D. 1982. Seasonality and phenology in a dry tropical forest in Ghana. Journal of Ecology 70:791-806.

LIMA, W.P.L. \& ZAKIA, M.J.B. 2000. Hidrologia de Matas Ciliares. In Matas ciliares: conservação e recuperação (R.R. Rodrigues \& H.F. Leitão Filho, eds.). Editora da USP/Fapesp, São Paulo, p.33- 44. 
MEDINA, E. \& KLINGE, H. 1983. Productivity of tropical forests and tropical woodlands. In Physiological plant ecology IV (O.L. Lange, P.S. Nobel, C.B. Osmond, \& H. Ziegler, eds.). Encyclopedia of Plant Physiology, vol. 12D, p.281-303.

MILLARD, P. 1995. Internal cycling of nitrogen in trees. Acta Horticulturae 383:3-14

MORELLATO, L.P.C. 1992a. Nutrient cycling in two southeast Brasilian forests. I - Litterfall and litter standing crop. Journal of Tropical Ecology 8:205-215.

MORELLATO, L.P.C. 1992b. História Natural da Serra do Japi: Ecologia e preservação de uma área florestal no sudeste do Brasil. Editora da Unicamp/Fapesp, Campinas.

MORELLATO, L.P.C., RODRIGUES, R.R., LEITÃO FILHO, H.F. \& JOLY, C.A. 1989. Estudo comparativo da fenologia de espécies arbóreas de floresta de altitude e floresta mesófila semidecídua na Serra do Japi, Jundiaí, São Paulo. Revista Brasileira de Botânica 12:85-98.

MORELLATO, L.P.C., TALORA, D.C., TAKAHASI, A., BENCKE, C.C., ROMERA, E.C. \& ZIPARRO, V.B. 2000. Phenology of Atlantic Rain Forest trees: a comparative study. Biotropica 32:811-823

NAIMAN, R.J. \& DÉCHAMPS, H. 1990. The ecology and management of aquatic-terrestrial ecotones. Man and the Biosphere series, vol. 4, Unesco, Parthenon Publ. Group, Paris.

NAIMAN, R.J., DÉCAMPS, H. \& FOURNIER, F. 1989. Role of land/inland water ecotones in landscape management and restoration: a proposal for collaborative research. MAB Digest 4, Unesco, Paris.

OLSON, J.S. 1963. Energy storage and the balance of producers and decomposers in ecological systems. Ecology 42:322-331

PAGANO, S.N. \& DURIGAN, G. 2000. Aspectos da ciclagem de nutrientes em Matas Ciliares do oeste do Estado de São Paulo, Brasil. In Matas ciliares: conservação e recuperação (R.R. Rodrigues \& H.F. Leitão Filho, eds.). Editora da USP/Fapesp, São Paulo, p.109-123
PEDRONI, F., SANCHEZ, M. \& SANTOS, F.A.M. 2002. Fenologia da copaíba (Copaifera langsdorffii Desf. Leguminosae, Caesalpinioideae) em uma floresta semidecídua no sudeste do Brasil. Revista Brasileira de Botânica 25:177-182.

RATHCKE, B. \& LACEY, E.P. 1985. Phenological patterns of terrestrial plants. Annual Review of Ecology and Systematics 16:179-214

RICHARDS, P.N. 1952. The tropical rain forest: an ecological study. Cambridge University Press, London.

RODRIGUES, R.R. \& GANDOLFI, S. 2000. Conceitos, tendências e ações para a recuperação de florestas ciliares. In Matas ciliares: conservação e recuperação (R.R. Rodrigues \& H.F. Leitão Filho, eds.). Editora da USP/Fapesp, São Paulo, p.235-247.

RODRIGUES, R.R. \& NAVE, A.G. 2000. Heterogeneidade florística das matas ciliares. In Matas ciliares: conservação e recuperação (R.R. Rodrigues \& H.F. Leitão Filho, eds.). Editora da USP/Fapesp, São Paulo, p.45-71

SALIS, S.M., TAMASHIRO, J.Y. \& JOLY, C.A. 1994. Florística e fitossociologia do estrato arbóreo de um remanescente de Mata Ciliar do Rio Jacaré-Pepira, Brotas, SP. Revista Brasileira de Botânica 17:93-103.

SALIS, S.M., SHEPHERD, G.J. \& JOLY, C.A. 1995. Floristic comparison of mesophytic semideciduous forests of the interior the state of São Paulo, Southeast Brazil. Vegetatio 119:155-164.

SÃO PAULO. 1989. Constituição do Estado de São Paulo. Imprensa Oficial. Assembléia legislativa do Estado de São Paulo. (http://www.al.sp.gov.br/index12.htm).

SWIFT, M.J., HEAL, O.W. \& ANDERSON, J.M. 1979. Decomposition in terrestrial ecosystems. $2^{\text {nd }} \mathrm{ed}$. University of California Press, Berkeley.

TANNER, E.V.J. 1981. The decomposition of leaf litter in jamaican montane rain forest. Journal of Ecology 69:263-275

ZAGATTO, E.A.G., JACINTO, A.O., REIS, B.F., KRUG, F.J., BERGAMIN FILHO, R., PESSENDA, L.C.R., MORTATTI, J. \& GINÉ, M.F. 1981. Manual de análises de plantas e águas, empregando sistemas de injeção em fluxo. Cena, USP, Piracicaba, São Paulo. 